investigation in larger more cohorts with more statistical power in order to improve CRC screening.

\begin{tabular}{ll}
$\begin{array}{l}\text { Abstract PTU-039 Table } 1 \\
\text { histopathological and endoscopic characteristics in missed CRC } \\
\text { cohort }\end{array}$ \\
\hline Anatomical site in colorectum (\% total) \\
\hline Sigmoid & $3(25 \%)$ \\
Splenic flexure & $1(8.33 \%)$ \\
Transverse & $3(25 \%)$ \\
Hepatic flexure & $2(16.67 \%)$ \\
Ascending & $2(16.67 \%)$ \\
Caecum & $1(8.33 \%)$ \\
& \\
Dysplasia (\% total) & $11(91.67 \%)$ \\
Low grade & $1(8.33 \%)$ \\
High grade & \\
\hline
\end{tabular}

\begin{tabular}{lll} 
Abstract PTU-039 Table 2 & CRC misses by endoscopist type \\
\hline Scope operator & $\begin{array}{l}\text { CRC misses (\% } \\
\text { total) }\end{array}$ & $\begin{array}{l}\text { Endoscopist miss rate (misses/total } \\
\text { colonoscopies) }\end{array}$ \\
\hline $\begin{array}{l}\text { Trainee } \\
\text { endoscopist }\end{array}$ & $3 / 12(25 \%)$ & $0.004(3 / 750)$ \\
$\begin{array}{l}\text { Surgical } \\
\text { Endoscopist }\end{array}$ & $2 / 12(16.67 \%)$ & $0.008(2 / 250)$ \\
$\begin{array}{l}\text { Physician } \\
\text { Endoscopist }\end{array}$ & $7 / 12(58.33 \%)$ & $0.00159(7 / 4400)$ \\
\hline
\end{tabular}

\section{PTU-040 HMGB1 IN THE PATHOGENESIS OF COLORECTAL CANCER}

Sandra Hapca*, Daniel P Brice, Susan Berry, Graeme I Murray, Mairi H McLean. School of Medicine, Medical Sciences and Nutrition, University of Aberdeen, Aberdeen, UK

\subsection{6/gutjnl-2018-BSGAbstracts.381}

Introduction High Mobility Group Box-1 (HMGB1) is a ubiquitous nuclear protein that regulates gene expression. When phosphorylated, it translocates to the cytoplasm and extracellular space to impact immune responses and epithelial cell behaviour. Our previous data revealed that dynamic subcellular localisation of HMGB1 is associated with colorectal neoplastic progression, with cytoplasmic HMGB1 expression a feature of early carcinogenesis. The leading edge of cancer invasion in polyp cancers $(\mathrm{CaP})$ had strong expression in both nuclear and cytoplasmic compartments. Our aim was to define the biological impact of this expression profile.

Methods $\mathrm{CD}^{+}$helper $\mathrm{T}$ cells, $\mathrm{CD}^{+}$cytotoxic $\mathrm{T}$ cells, $\mathrm{FOXP}^{+}$regulatory $\mathrm{T}$ cells, $\mathrm{CD} 20^{+} \mathrm{B}$ cells and $\mathrm{CD}^{+} 8^{+}$macrophages were assessed by immunohistochemistry on endoscopically retrieved paraffin embedded $\mathrm{CaP}$ lesions sourced from the Grampian Tissue Biorepository $(n=25)$. Ethical approval was granted by the Grampian Biorepository Scientific Access Group. The invasive cancer margin was identified by a gastrointestinal pathologist. The immune cell infiltrate was expressed as number of positive cells per high power field (x20) in the adjacent stroma alongside intensity of nuclear and cytoplasmic HMGB1 expression. The impact of HMGB1 on colonic epithelial cell behaviour was assessed by stimulation of Caco-2 cells (ATCC HTB-37TM) for 48-72 hours with 0, 50 and $100 \mathrm{ng} / \mathrm{ml}$ recombinant HMGB1 with (1) analysis of wound healing by scratch assay (images captured at time 0 and 24 hour intervals and analysed with ImageJ), (2) proliferation by incorporation of tritiated thymidine and (3) expression of permeability genes (CLDN2, CLDN4, OCLN, CDH1, TJP1) using TaqMan RT-qPCR, normalised to expression of GAPDH and $B 2 M$ and analysed by the Livak method.

Results A robust inflammatory infiltrate was identified adjacent to the invasive cancer margin with a preponderance of $\mathrm{CD} 4^{+}$ $\mathrm{T}$ cells and $\mathrm{CD}^{+} 8^{+}$macrophages. The subcellular localisation of HMGB1 (nuclear versus cytoplasmic) did not result in phenotypically distinct populations. There was no difference in epithelial restitution in response to HMGB1 over 48 hours, although high concentration at 72 hours was associated with reduced wound healing $(\mathrm{p}<0.01)$ and reduced proliferation rate $(\mathrm{p}<0.01)$. HMGB1 stimulation significantly decreased the expression of CLDN4 ( $<<0.008)$.

Conclusions Cytoplasmic HMGB1 expression did not impact the phenotype of adjacent immune cell infiltrate at the cancer margin. HMGB1 influences growth dynamics of colonic epithelial cells and reduces the expression of the pore closing tight junction gene, CLDN4. Understanding HMGB1 driven biology in $\mathrm{CaP}$ could identify an important mechanism for early carcinogenesis.

\section{PTU-041 METHANOGENESIS IN THE GI TRACT - IMPLICATIONS FOR UK BREATH TESTING}

Jordan Haworth*, Jennifer Haynes, Charlotte Pitcher, Sam Treadway, Anthony Hobson. The Functional Gut Clinic, Manchester, UK

\subsection{6/gutjnl-2018-BSGAbstracts.382}

Introduction Methane production from gut microbes has been implicated in clinical constipation and manipulation of the microbiome represents an attractive and alternate therapeutic target. Methane production is not routinely measured during breath testing but the recent North American Consensus (NAC) document has recommended this as standard. To assess prevalence of methanogenesis in patients presenting with bloating symptoms we performed a retrospective analysis of our breath test database from the preceding 6 months.

Methods In total 736 subjects were analysed. The presence of methane levels $\geq 10$ ppm was considered methane-positive. Breath tests for Carbohydrate Malabsorption and Small Intestinal Bacterial Overgrowth (SIBO) were included. Baseline and intra-study bloating was scored on a scale $0-10$ with 0 being absent and 10 being extreme. Results were analysed statistically using independent t-tests.

Results 136 subjects (18.5\%) had a positive result for excessive methane. Within this group,129 subjects had a SIBO breath test with $64.3 \%$ having a negative result. The remaining $35.7 \%$ had excessive methane and SIBO (determined by a rise in hydrogen $>10 \mathrm{ppm}$ above baseline within $60 \mathrm{~min}$ after ingestion of substrate). There was an overall increase in bloating symptoms for methane-positive compared to methane negative patients $(p=0.035)$ in the absence of SIBO.

Discussion Changes in the microbiome to a methane predominant environment occurs in about $20 \%$ of patients presenting with bloating symptoms. This supports the NAC statement that methane should be measured routinely during breath 